NUSC Technlcal Report $83 i 7$

1 June 1988

\title{
The Wigner Distribution Function With Minimum Spread
}

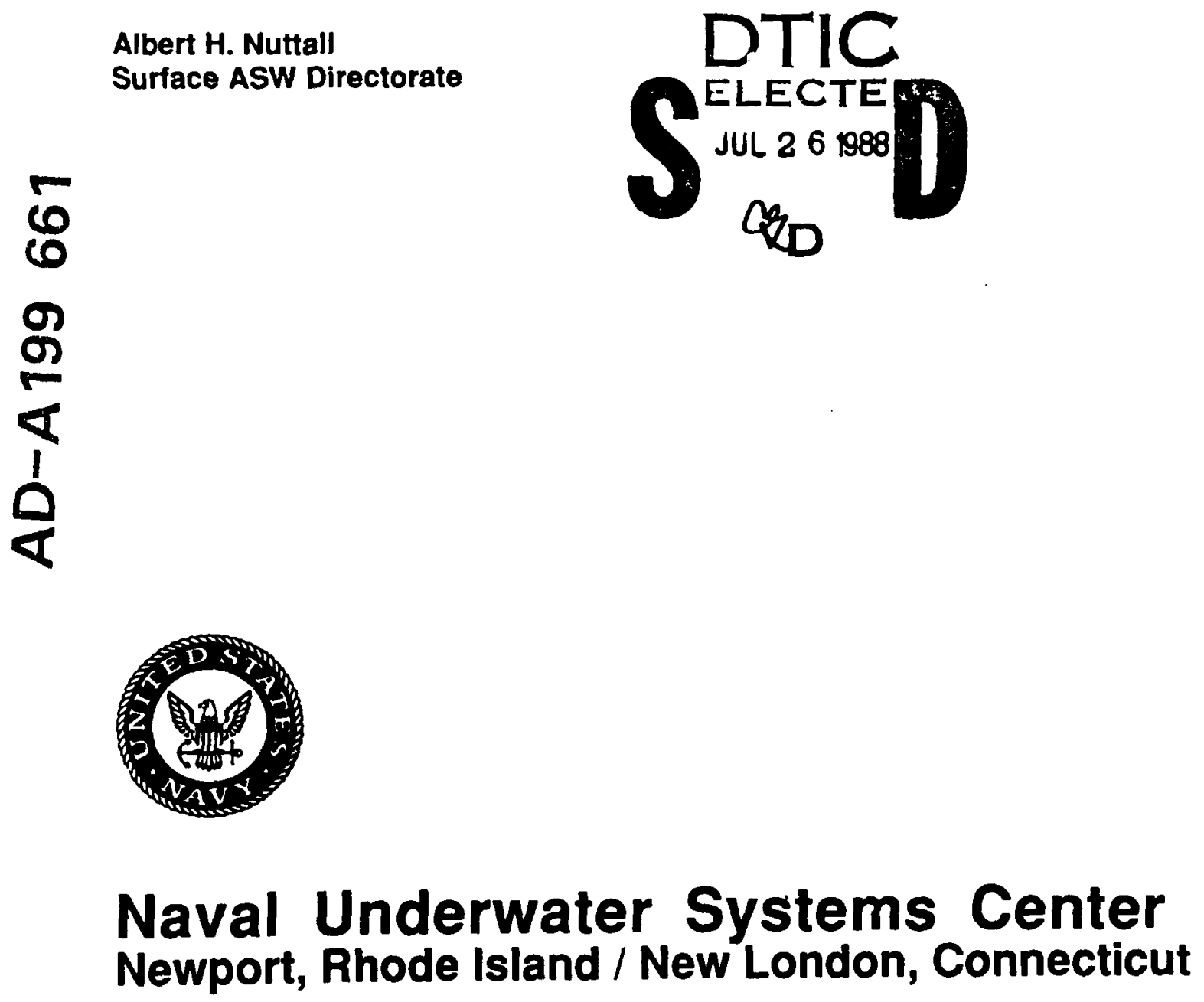

Approved for public release; distribution is uniimlted. 
UNCLASS IFIED

SECUUAIN CLASSIFACATION OF THIS PAUE

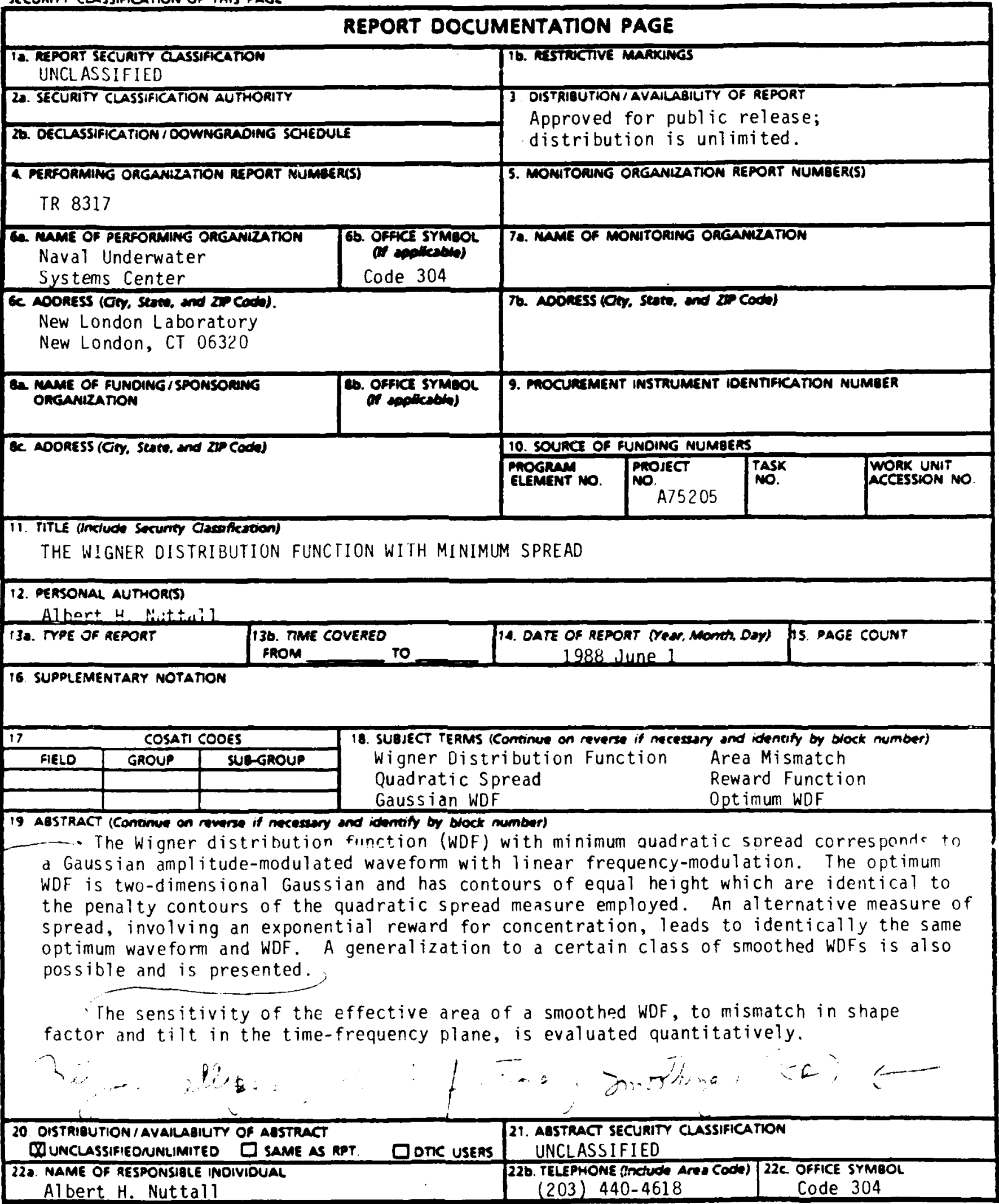

DO FORM 1473, B4 MAR

o3 APh edition may be used until exhausted. All other editions are obsolete.
SECUAITY CLASSIFICATION OF THIS PAGE UNCLASS I FIED 
INICLASSIFIED

SECURITY CLAHSIFICA TIOM OP THIS PAOE

18. SUBJECT TERMS (Cont'd.)

Short-Term Spectral Estimate

Smoothing

Two-Dimensional Convolution

Concentrated WDF 
LIST OF ILLUSTRATIONS . . . . . . . . . . . . . . . . . . . . . . . .

LIST OF SYMBOLS. . . . . . . . . . . . . . . . . . . . . .

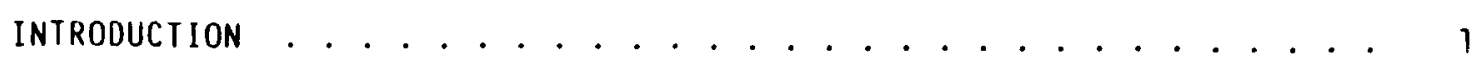

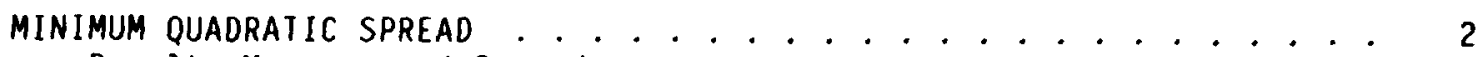

Penalty Measure and Spread .................. . . . . 3

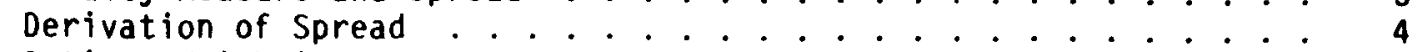

Optimum Weighting..................... 6

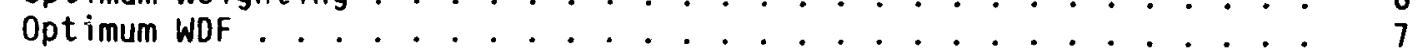

ALteRnATIVE REWARD MEASURE ....................... . . . 8

GENERAL.IZATION TO SMOOTHED WOF ...................... . . . 9

SENSITIVITY TO MISMATCH ....................... . . . 12

SUMMARY . . . . . . . . . . . . . . . . . . . . . . . 17

APPENDIX A. MaXimization OF Reward VAlUe ................ 18

APPENDIX B. MOMENTS OF DISTRIBUTION D . . . . . . . . . . . . . . . . 27

APPENDIX C. GENERAL TILTED ElLIPSE . . . . . . . . . . . . . . . . . . 31

APPENDIX 0 . KERNEL APPROACH TO PENALTY FUNCTION . . . . . . . . . . 36

REFERENCES ............................. 40

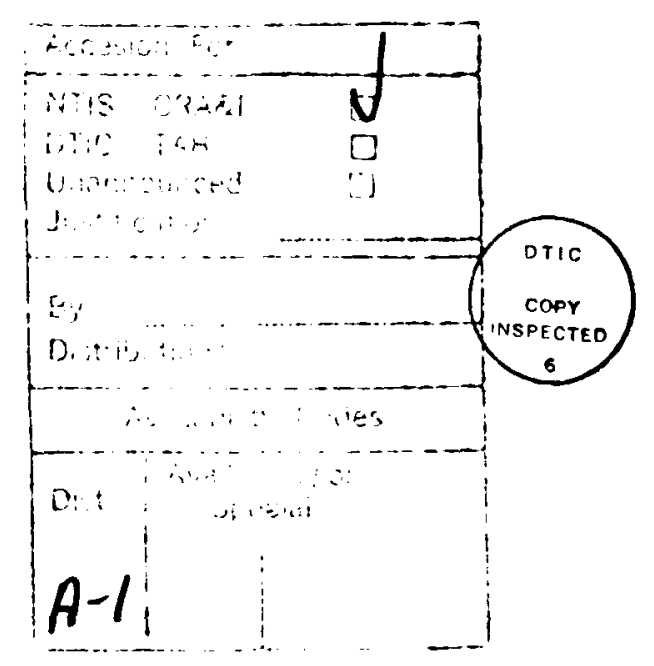


TR 8317

\section{LIST OF ILLUSTRATIONS}

$\begin{array}{ll}\text { Figure } & \text { Page }\end{array}$

1 Contour Ellipses for Mismatched Tilt........... 15

2 Area Ratio (34) for $F_{1}=2, F_{2}=2, A_{2}=2, B_{1}=\pi / 4 \ldots . . .15$

3 Contour Ellipses for Mismatched Shape Factor......... 16

4 Area Ratio (34) for $F_{1}=2, A_{2}=2, B_{1}=\pi / 4, B_{2}=\pi / 4 . . . .16$

C-1 Rotated Coordinate Axes ............... 31

\section{LIST OF SYMBOLS}

$\begin{array}{ll}\text { WOF } & \text { Wigner distribution function } \\ t & \text { time } \\ f & \text { frequency } \\ s(t) & \text { waveform analyzed, (1) } \\ u(t) & \text { weighting function, (1) } \\ S_{u} & \text { short-term spectral estimate, (1) } \\ W_{S} & \text { WDF of } s(t),(1) \\ W_{U} & \text { woF of } u(t),(1),(2) \\ \otimes & \text { convolution, (1) } \\ \delta & \text { delta function } \\ P(t, f) & \text { penalty measure, (3) } \\ a, b, c & \text { parameters of } P,(3) \\ I & \text { spread of wDF } W_{U},(4)\end{array}$




\section{LIST OF SYMBOLS (Cont'd)}

\begin{tabular}{|c|c|}
\hline 0 & real constant, (5) \\
\hline$u^{\prime}(t)$ & derivative of $u(t)$ with respect to $t,(10)$ \\
\hline B & complex constant, (11) \\
\hline$T(t)$ & temporary auxiliary function, (13) \\
\hline$u_{0}(t)$ & optimum weighting, (20) \\
\hline$I_{0}$ & minimum spread, (21) \\
\hline$w_{0}$ & opt imum WDF, (22) \\
\hline 0 & generalized smouthing distribution, (23) \\
\hline$v_{2}$ & smoothing function, (23) \\
\hline$v$ & frequency separation variable, (23), (24) \\
\hline$\tau$ & time separation variable, (23), (24) \\
\hline$\chi_{u}$ & complex ambiguity function of $u,(24)$ \\
\hline$q_{2}$ & double Fourier transform of $v_{2}$, (25) \\
\hline superscript $v$ & partial derivative with respect to $v$, (27) \\
\hline superscript $\tau$ & partial derivative with respect to $\tau$, (27) \\
\hline $\mathrm{I}_{\mathrm{D}}$ & spread of distribution $0,(30)$ \\
\hline$R(t, f)$ & reward function, $(22 A),(A-1)$ \\
\hline$v$ & reward value, $(22 B),(A-5)$ \\
\hline$K(x, y)$ & Hermitian kernel, $(A-11),(A-12)$ \\
\hline$\lambda_{n}$ & eigenvalue, $(A-12)$ \\
\hline$\sigma_{n}(x)$ & eigenfunction, $(A-12)$ \\
\hline$H e_{n}(x)$ & Hermite polynomial, $(A-27)$ \\
\hline A & area of ellipse, figure $c-1$ \\
\hline B & tilt of ellipse, figure $\mathrm{C}-1$ \\
\hline$F$ & shape factor of ellipse, $(C-4)$ \\
\hline
\end{tabular}


THE WIGNER DISTRIBUTION FUNCIION

WITH MINIMUM SPREAD

\author{
INTRODUCTION
}

\begin{abstract}
A number of advantageous features associated with smoothing a wigner distribution function (WOF) were discussed in a recent report [1]. At that time, it was shown that the WDF with minimum quadratic spread, about the line $t=B_{c} t$ in the time-frequency plane, was a two-dimensional Gaussian function, when constraints of finite energy and mean-square duration were imposed [1, app. G]. However, a more appropriate measure of spread about the origin in the $t, f$ plane is adopted here and minimized, yielding a unique waveform and corresponding WDF. Additionally, a reward measure for concentration is shown to yield identically the same optimum wDF.
\end{abstract}

An additional property of smoothing two-dimensional WDFs was also demonstrated; namely, if two Gaussian mountains are doubly-convolved with each other, the effective area of the result is greater than the sum of the two effective areas, unless the contours of both woFs have the same tilt and ratio of major-to-minor axes [1, app. J]. A quantitative investigation of the effect of mismatch in these parameters on the effective area is conducted herein.

It is assumed that the reader is familiar with the content and approach of the earlier report; accordingly, this follow-on effort will be briefer and will not review the considerable history and background of the woF. 
It was shown in $[1,(102)$ and (106)] that the short-term spectral estimate is equal to the double convolution of the WOF of the waveform $s(t)$ being analyzed with the wof of the weighting $u(t)$ employed. That is,

$$
\begin{aligned}
& \left|s_{u}(t, f)\right|^{2} \equiv\left|\int d t_{1} \exp \left(-i 2 \pi f t_{1}\right) s\left(t_{1}\right) u^{\star}\left(t-t_{1}\right)\right|^{2}= \\
& =\iint d t_{1} d f_{1} w_{s}\left(t_{1}, f_{1}\right) w_{u}\left(t-t_{1}, f-f_{1}\right)=w_{s}(t, f) \circledast w_{u}(t, f),
\end{aligned}
$$

where $\otimes$ denotes convolution. Here,

$$
W_{u}(t, f)=\int d \tau \exp (-i 2 \pi f \tau) u\left(t+\frac{\tau}{2}\right) u^{\star}\left(t-\frac{\tau}{2}\right)
$$

is the WDF of complex weighting $u(t)$; a similar definition holds for wDF $W_{S}$. Generalizations to non-wigner smoothing functions for $W_{u}$ are given in $[1$, app. F].)

Since the WDF $W_{s}$ of waveform $s(t)$ has some good energy localization properties (and some deleterious negative oscillations), it is desired that the smearing in the $t, f$ plane, implied by convolution (1), be minimized. That is, we would like WDF $W_{u}$ of weighting $u(t)$ to be as concentrated as possible about the origin of the $t, f$ plane. The ideal of an impulse, $\delta(t) \delta(f)$, is not a legal WDF, and must he discarded. Since the left-hand side of (1) can never be negative, we can be assured, by this smoothing procedure of two WDFs, that we will always get a physically-meaningful distribution in the t,f plane: that is, the smoothed distribution will always be non-negative for all $t, f$ and have a volume equal to the energy of waveform $s(t)$. For example, see [1, (111) et seq.]. 
PENALTY MEASURE AND SPREAD

In order to confine WOF $W_{u}$ near the origin, we define a penalty measure which is zero at $t, f=0,0$ and which increases quadratically with $t$ and $f$. Namely, the penalty measure is

$$
P(t, f)=a^{2} t^{2}+4 \pi^{2} b^{2} f^{2}+4 \pi c t f, a, b, c \text { real, }
$$

and the corresponding spread of the WDF $W_{u}$ is defined as

$$
I=\iint d t d f w_{1}(t, f) P(t, f) \text {. }
$$

Contours of equal penalty in (3) are tilted ellipses in the $t . f$ plane; these would be selected upon observation of a calculated WDF $W_{s}$ of waveform $s(t)$ in regions of interest, i.e., high activity.

Therefore, real constants $a, b, c$ are presumed known. Define quantity

$$
0=a^{2} b^{2}-c^{2}
$$

Then, in order that penalty

$$
P(t, f)>0 \text { for } t, f \neq 0,0 \text {. }
$$

it is necessary that

$$
Q>0 \text {. }
$$

The property (6) was not satisfied by penalty furiction $\left(f-\beta_{c} t\right)^{2}$ in [1, app. 6]; that function was zero all along the line $f=B_{c} t$, allowing the WDF to become impulsive there. 
We also want WOF $W_{u}$ in (1) and (4) to have unit volume, for two reasons. First of all, this will guarantee that the st.ort-term spectral estimate on the left-hand side of (1) will have a volume equal to the signal energy, regardless of weighting $u(t)$ employed. Secondly, without this volume constraint, $u(t)$ and $w_{u}$ would collapse to zero, giving a meaningless spread value of $I=0$ in (4). Thus we require that

$$
1=\iint d t d f w_{u}(t, f)=\int d t|u(t)|^{2}
$$

Subject to this integral constraint, we want to minimize spread I in (4), and find the particular weighting $u(t)$ and corresponding opt imum wOF $W_{u}$. Notice that we are imposing no constraint of positivity on $w_{u}$.

DERIVATION OF SPREAD

Substitute (3) into (4) to get spread

$$
I=\iint d t d f w_{u}(t, f)\left(a^{2} t^{2}+4 \pi^{2} b^{2} f^{2}+4 \pi c t f\right) .
$$

where WDF $W_{u}$ is given in terms of $u(t)$ according to (2). By using the results in $[1,(G-4)$ and $(G-5)]$, we can express (9) solely in the time domain as

$$
\begin{aligned}
I & =a^{2} \int d t t^{2}|u(t)|^{2}+b^{2} \int d t\left|u^{\prime}(t)\right|^{2}+2 c \int d t \operatorname{Im}\left\{u^{\prime}(t) u^{\star}(t)\right\}= \\
& =\int d t\left[a^{2} t^{2}|u(t)|^{2}+b^{2}\left|u^{\prime}(t)\right|^{2}+i c t u(t) u^{\prime \star}(t)-\operatorname{ict} u^{\star}(t) u^{\prime}(t)\right] .
\end{aligned}
$$

For reasons to become apparent shortly, define complex constant

$$
B=\frac{\sqrt{a}+i c}{b^{2}}
$$


TR 8317

then, by using (5), we find

$$
b^{2}|B|^{2}=a^{2}
$$

Now consider the quantity

$$
\begin{gathered}
T(t) \equiv b^{2}\left|u^{\prime}(t)+B t u(t)\right|^{2}= \\
=b^{2}\left|u^{\prime}(t)\right|^{2}+b^{2}|B|^{2} t^{2}|u(t)|^{2}+b^{2} B t u(t) u^{\prime \star}(t)+b^{2} B^{\star} t u^{\star}(t) u^{\prime}(t)= \\
=b^{2}\left|u^{\prime}(t)\right|^{2}+a^{2} t^{2}|u(t)|^{2}+(\sqrt{D}+i c) t u(t) u^{\prime \star}(t)+ \\
+(\sqrt{D}-i c) t u^{\star}(t) u^{\prime}(t) .
\end{gathered}
$$

Comparison of (10) and (13) immediately reveals that

$$
\int d t T(t)=I+\sqrt{Q} \int d t t\left[u(t) u^{\prime \star}(t)+u^{\star}(t) u^{\prime}(t)\right] .
$$

We now integrate by parts, letting

$$
U=t u(t), d V=d t u^{* *}(t)
$$

to find that

$$
\begin{aligned}
& \int d t \operatorname{t} u(t) u^{\prime \star}(t)=-\int d t\left[u(t)+t u^{\prime}(t)\right] u^{\star}(t)= \\
& =-\int d t|u(t)|^{2}-\int d t t u^{\prime}(t) u^{\star}(t) .
\end{aligned}
$$

We presume that $u(t)$ goes to zero at $t= \pm \infty$, consistent with energy constraint (8).

When (16) is employed in (14), there follows

$$
\int d t T(t)=1-\left.\sqrt{Q} \int d t \mu(t)\right|^{2} .
$$

5 
Thus the desired expression for spread I is given by (17) and (13) as

$$
I=b^{2} \int d t\left|u^{\prime}(t)+B t u(t)\right|^{2}+\sqrt{Q} \int d t|u(t)|^{2}
$$

This general result holds for any weighting $u(t)$; it is obviously positive in all cases, since $0>0$.

OPTIMUM WEIGHTING

The last term in (18) cannot be altered; it is equal to $\sqrt{0}$, as seen by reference to constraint ( 8 ). Furthermore, the minimum value for the remaining term in (18) is zero and is obtained for weighting $u(t)$ which satisfies the differential equation

$$
u^{\prime}(t)+B t u(t)=0 \text { for all } t \text {. }
$$

The only solution to (19) is

$$
u_{0}(t)=A \exp \left(-\frac{1}{2} B t^{2}\right) \text { for all } t
$$

where complex constant $A$ is chosen for unit energy, and $B$ is given by (1?). That is, $u_{0}(t)$ has Gaussian amplitude-modulation and linear frequencymodulation. The phase of $A$ is ambiguous.

The resuitant minimum value of spread $I$ in $(18)$ is obviously

$$
I_{0}=\sqrt{a}=\sqrt{a^{2} b^{2}-c^{2}}
$$

where we employed (5). It is always positive, as seen by reference to requirements (6) and (7). 
TR 8317

OPIIMUM WDF

The WDF corresponding to optimum weighting $(20)$ is obtained by substitution in (2), and use of $[1,(\mathrm{H}-17)$ and $(\mathrm{H}-18)]$, as

$$
w_{0}(t, f)=2 \exp \left[-\frac{a^{2} t^{2}+4 \pi^{2} b^{2} f^{2}+4 \pi c t f}{\sqrt{0}}\right] \text {. }
$$

The area of the contour ellipse at the $1 / \mathrm{e}$ relative level $151 / 2$ in the $t, f$ plane, as expected.

Observe that the numerator of the exp in (22) is identically the quadratic penalty function $P(t, f)$ imposed in (3). That is, the contours of optimum WDF (22) are identical to the contours of equal penalty of $P(t, f)$ in (3). This result is intuitively satisfying: the optimum wOF packs as much volume inside a given penalty contour as possible, to the extent that the resultant WDF values are equal all along that given penalty contour.

Observe also, that although positivity of the WDF $W_{u}$ was not imposed as a constraint in the minimization of spread I in (4) or (9), the resultant optimum WOF in (22) is, in fact, everywhere positive. Although the optimum weighting (20) has an ambiguous phase, the optimum WDF has no ambiguity; there is a unique optimum WDF, namely (22). 


\section{ALTERNATIVE REWARD MEASURE}

Instead of penalizing the spread of wOF $w_{u}$ about the origin in $t, f$ space, we could alternatively utilize a measure which rewards concentration about $t, f=0,0$. In particular, consider reward function

$$
R(t, f)=\exp \left[-a^{2} t^{2}-4 \pi^{2} b^{2} f^{2}-4 \pi c t f\right]
$$

and reward value

$$
V=\iint d t d f R(t, f) w_{u}(t, f)
$$

for WOF $W_{u}$. The origin value of $R(t, f)$ is 1 ; in order for $R(t, f)$ to decay to zero as $t$ and/or $f$ tend to infinity, we must have condition (7) satisfied again. Notice that the contours of equal reward are ellipses in the $t, f$ plane.

The maximization of reward value $V$, subject to volume constraint ( 8 ) on $W_{u}$, is conducted in appendix $A$. It is shown there that the optin. Im weighting is again (20), and that the optimum WDF is (22). The maximum value of reward $V$ is

$$
v_{\max }=\frac{1}{1+\sqrt{0}}=\frac{1}{1+\sqrt{a^{2} b^{2}-c^{2}}} .
$$

More general results, for arbitrary reward functions $R(t, f)$ in $(22 b)$, are presented in appendix $A$. 
A general class of distributions* has been presented in $[2,(1.7)$ and $(1.8)]$. In current notation, that class is given by $[1,(F-1)]$ as

$$
\begin{aligned}
& D(t, f) \equiv w_{u}(t, f) \stackrel{t f}{\circledast} v_{2}(t, f)= \\
& =\iint d v d \tau \exp (i 2 \pi v t-i 2 \pi f \tau) \chi_{u}(v, \tau) a_{2}(v, \tau),
\end{aligned}
$$

where WOF $W_{u}$ is given by $(2)$, and $v_{2}(t, f)$ is a general two-dimensional smoothing function. The complex ambiguity function of $u(t)$ is

$$
\chi_{u}(v, \tau)=\int d t \exp (-i 2 \pi v t) u\left(t+\frac{\tau}{2}\right) u^{\star}\left(t-\frac{\tau}{2}\right) .
$$

while

$$
a_{2}(v, \tau)=\iint d t d f \exp (-i 2 \pi v t+i 2 \pi f \tau) v_{2}(t, f)
$$

is a double fourier transform of the smoothing function $v_{2}$. Observe that if there is no smoothing, then

$$
\begin{gathered}
v_{2}(t, f)=\delta(t) \delta(f) \\
q_{2}(v, \tau)=1 \text { for all v, } \tau \\
D(t, f)=w_{u}(t, f) .
\end{gathered}
$$

*This section is based upon a suggestion by Leon Cohen. Hunter College, New York. NY, that the optimum WDF results here actually apply to a wider class of distributions. 
Now it is shown in appendix $B$ that the following second morients ui generalized smoothing distribution $D$ can te expressed in terms of derivatives of $\chi_{u}$ and $a_{2}$ at the origin:

$$
\begin{aligned}
& \iint d t d f t^{2} D(t, f)=-\frac{1}{4 \pi^{2}}\left[\chi_{u}^{v v}(0,0) a_{2}(0,0)+2 \chi_{u}^{v}(0,0) q_{2}^{v}(0,0)+\right. \\
& \left.+\chi_{u}(0,0) q_{2}^{v v}(0,0)\right], \\
& \iint d t d f t f D(t, f)=\frac{1}{4 \pi^{2}}\left[\chi_{u}^{\tau v}(0,0) a_{2}(0,0)+\chi_{u}^{\tau}(0,0) q_{2}^{v}(0,0)+\right. \\
& \left.+\chi_{u}^{v}(0,0) q_{2}^{\tau}(0,0)+\chi_{u}(0,0) q_{2}^{v \tau}(0,0)\right], \\
& \iint d t d f f^{2} 0(t, f)=-\frac{1}{4 \pi^{2}}\left[\chi_{u}^{\tau \tau}(0,0) a_{2}(0,0)+2 \chi_{u}^{\tau}(0,0) q_{2}^{\tau}(0,0)+\right. \\
& \left.+\chi_{u}(0,0) q_{2}^{\tau \tau}(0,0)\right] .
\end{aligned}
$$

Here, for example, superscript $v$ denotes a partial derivative with respect to $v$, which is then evaluated at the origin $v, \tau=0,0$.

If follows imnediately that if origin value

$$
q_{2}(0,0)=1
$$

and if the five origin derivatives

$$
q_{2}^{v}(0,0)=q_{2}^{\tau}(0,0)=q_{2}^{v v}(0,0)=q_{2}^{v \tau}(0,0)=q_{2}^{\tau \tau}(0,0)=0 .
$$

then (27) reduces to the moments that would have resulted from employing the no-smoothing result (26) in (27). Thus, distributions $D(t, f)$ resulting from (23), with properties (28) and (29) for $q_{2}$, have the same second moments 
TR 8317

as the WDF $W_{u}(t, f)$. Hence, the spread $I_{D}$ of distribution $D(t, f)$ is given by (see $(9))$

$$
\begin{aligned}
I_{D} & =\iint d t d f D(t, f)\left(a^{2} t^{2}+4 \pi^{2} h^{2} f^{2}+4 \pi c t f\right)= \\
& =\iint d t d f W_{u}(t, f)\left(a^{2} t^{2}+4 \pi^{2} b^{2} f^{2}+4 \pi c t f\right)=I,
\end{aligned}
$$

which is exactly the spread I of WOF $W_{u}(t, f)$. That is, smoothed distribution $D(t, f)$ in (23) has the same $\operatorname{spread}$ as $W D F w_{u}(t, f)$, when smoothing function $v_{2}(t, f)$ (actually transform $q_{2}$ ) satisfies the properties in (28) and (29). Notice that these properties are considerably less restrictive than requiring

$$
a_{2}(v, 0)=a_{2}(0, \tau)=1 \text { for all } v, \tau \text {. }
$$

which arises when one is interested in maintaining the marginals $[2,(1.6)]$.

We must also observe from (23) that the volume under generalized smoothing distribution $D$ is equal to the product of the volume under $w_{u}$ and the volume under $v_{2}$. But the latter quantity is unity, by virtue of (28). What all this means is, that if we minimize spread $I_{D}$ in $(30)$, subject to a unit volume constraint on 0 , the end result is precisely (18) and (20), and the optimum WDF $W_{u}$ is again given by (22). The corresponding distritution $D$ is obtained by substitution of (22) into (23) and specification of the complete $v_{2}$ or $a_{2}$ functions. The properties in (28) and (29) are not sufficient to completely specify $a_{2}$ or 0 ; all that is specified by (28) and (29) are the second order moments of $D$ in (27). It should also be noted that all the conditions in (29) cannot be met by the general tilted Gaussian $a_{2}$ function employed in $[1,(F-9)$ and seque 1 to $(F-12)]$. 
TR 8317

\section{SENSITIVITY TO MISMATCH}

In [1, app. J], it was shown that if two Gaussian mountains are doubly-convolved in $x, y$ space, the effective area $A_{3}$ of the resultant is greater than the sum of the individual areas, except when the two elliptical contours have the same tilt and the same ratio of major-to-minor axis (shape factor). Here, we wish to investigate, quantitatively, the increase in effective area above the minimum value, when the tilt and shape factors are not at their optimum values. This situation can arise when observation of WDF $W_{s}$ of waveform $s(t)$ is contaminated, in a particular region of interest in the $t, f$ plane, by interference effects, thereby making estimation of the tilt and the shape factor of the elliptical cortours somewhat inaccurate.

The general situation is considered mathematically in appendix $C$. Ellipse 1 has

$$
\text { area } A_{1} \text {, tilt } B_{1} \text {, shape factor } F_{1} \text {, }
$$

while ellipse 2 has

$$
\text { area } A_{2} \text {, tilt } B_{2} \text {, shape factor } F_{2} \text {. }
$$

The ratio

$$
\frac{A_{3}}{A_{1}+A_{2}}
$$

is presented in $(C-13)$ in terms of a number of auxiliary quantities. 
The initial example we consiaer is where ellipse 1 has seven different areas, namely

$$
A_{1}=.5,1,2,3,4,5,6 \quad B_{1}=\frac{\pi}{4} \quad F_{1}=2 .
$$

The tilt is fixed at $\pi / 4$ radians and the shape factor at 2 . On the other hand, elipse 2 has

$$
A_{2}=2 \quad B_{2}=-\frac{\pi}{4} \text { to } \frac{\pi}{4} \quad F_{2}=2 .
$$

That is, the shape factor is perfect at $F_{2}=F_{1}=2$, but the tilt is Sweit over a $\pi / 2$ range (greater discrepancies than $\pi / 2$ lead to obvious periodicities and symmetries centered about $B_{2}=B_{1}$ as well as about $\beta_{2}=\beta_{1} \pm \pi / 2$ and about $\left.\beta_{2}=\beta_{1}+\pi\right)$. The situation under investigation is depicted in figure 1, where ellipse 2 is dotted.

The effect of mismatch in tilt is presented quantitatively in figure 2. As expected, ratio $(34)$ is 1 at $B_{2}=B_{1}=\pi / 4$, regardless of area $A_{1}$. The most degradation (upper-most curve) is realized for $A_{1}=2$, i.e., when the areas of the two ellipses are equal. The maximum increase in area is only 25 percent, when $B_{2}$ is off by $\pi / 2$ radians; however, if the shape factor is significantly larger than 1, the sensitivity to the tilt would be much greater, as figure 1 shows.

The final example utilizes the exact same parameter values as (35) for ellipse 1, while ellipse 2 has

$$
A_{2}=2 \quad B_{2}=\frac{\pi}{4} \quad F_{2}=2 \text { to } 6 .
$$


TR 8317

Now the tilt is perfect at $B_{2}=B_{1}=\pi / 4$, but the shape factor $F_{2}$ varies above the best value of 2 . The situation is depicted in figure 3 , where ellipse 2 is again dotted.

Ratio (34) is plotted in figure 4 versus the shape factor $F_{2}$. Again, the upper-most curve corresponds to the case where $A_{1}=A_{2}=2$. There is no need to compute ratio (34) for $F_{2}<F_{1}=2$, because the values for $F_{2}=F_{1} r$ are the same as those for $F_{2}=F_{1} / r$. Additional cases of interest can be investigated by use of the program listed in appendix $C$. 


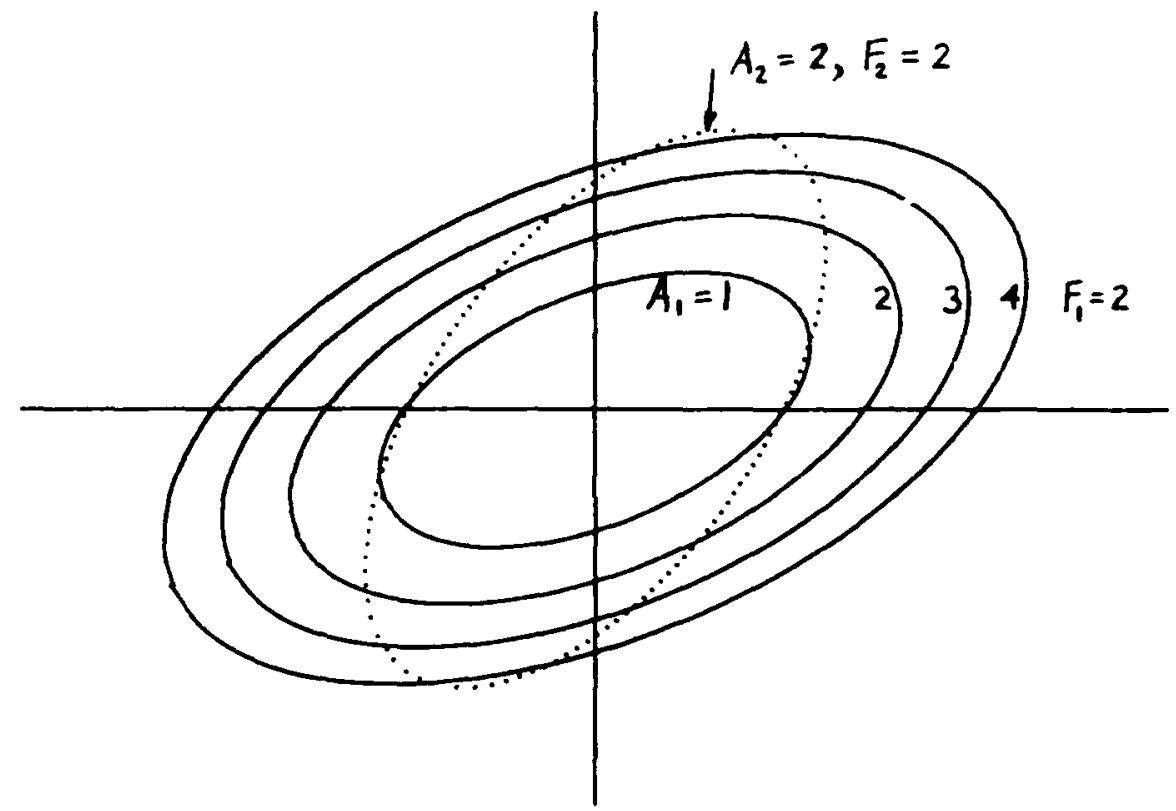

Figure 1. Contour Ellipses for Mismatched Tilt

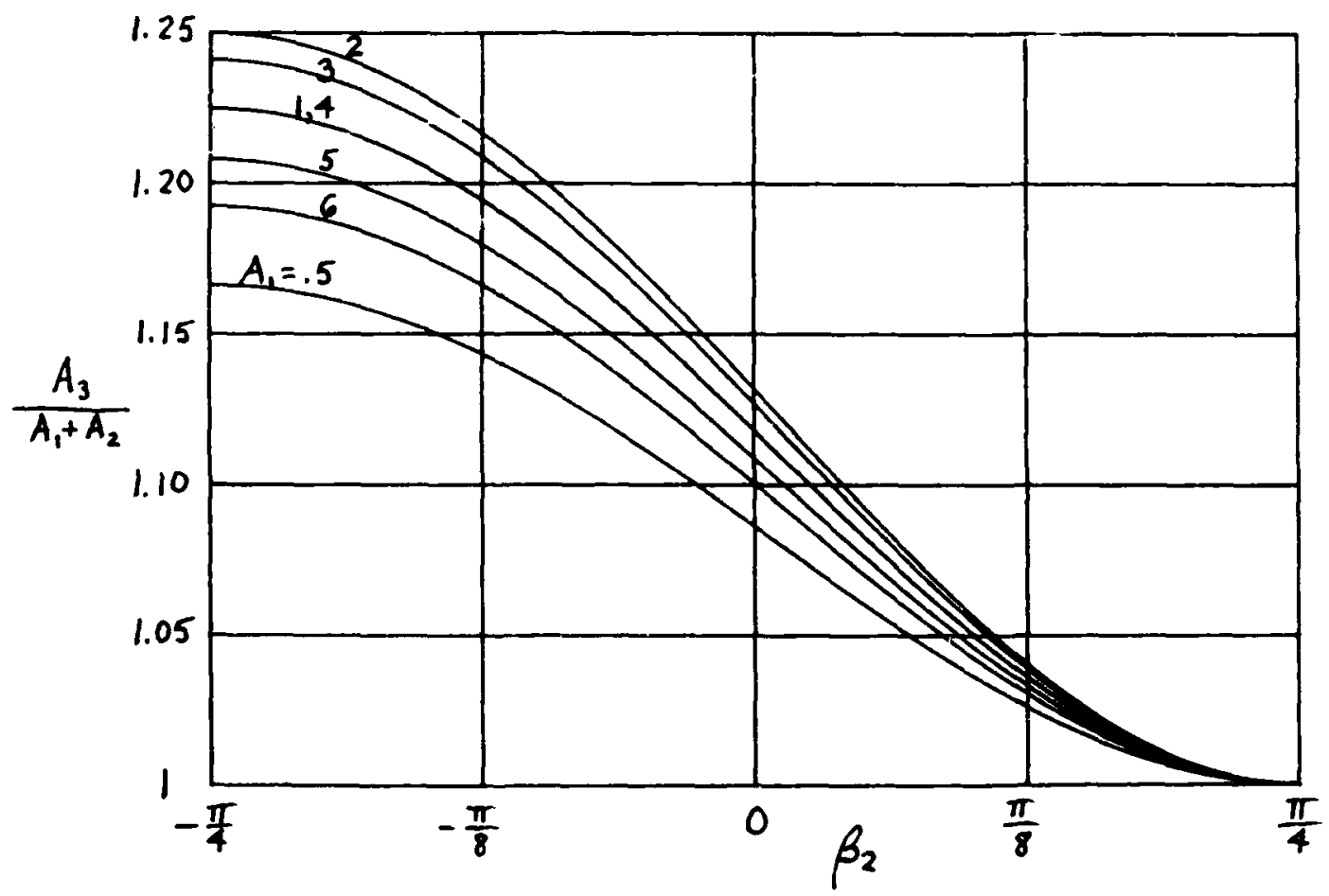

Figure 2. Area Ratio (34) for $F_{1}=2, F_{2}=2, A_{2}=2, \beta_{1}=\pi / 4$ 
TR 8317

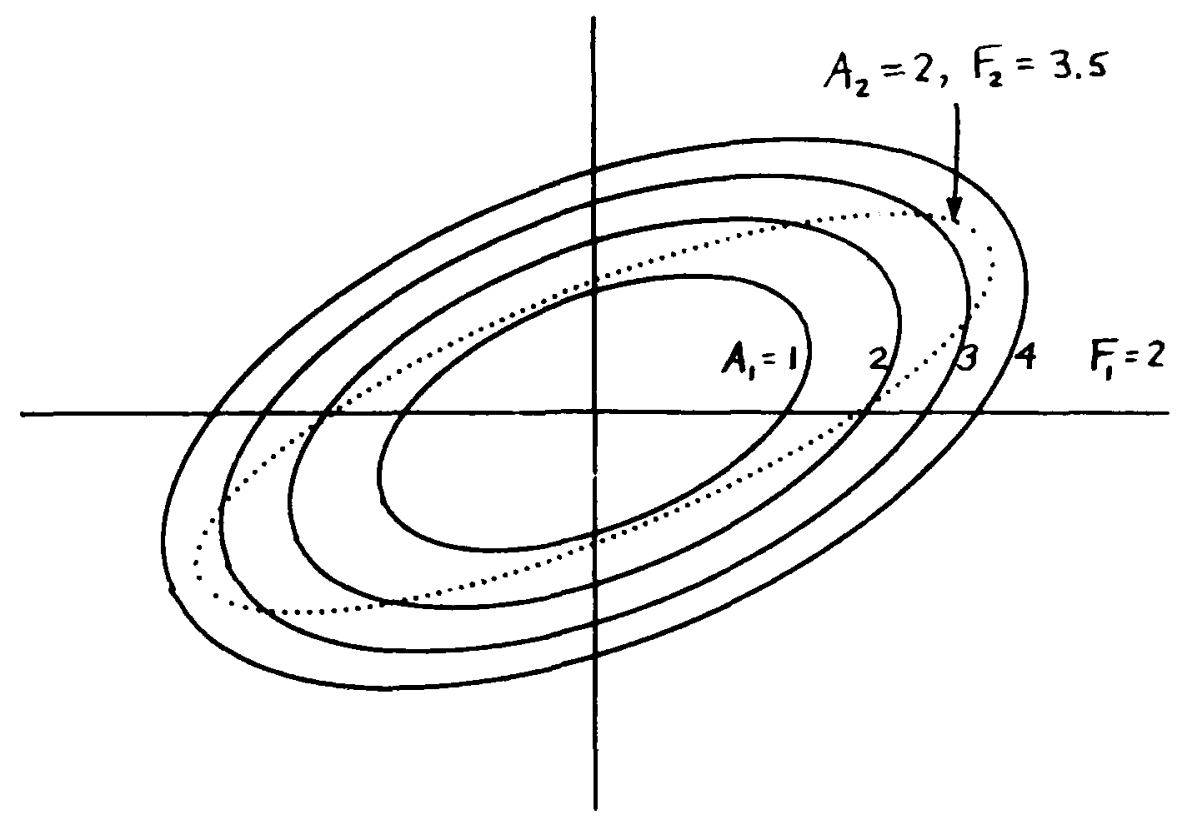

Figure 3. Contour Ellipses for Mismatched Shape Factor

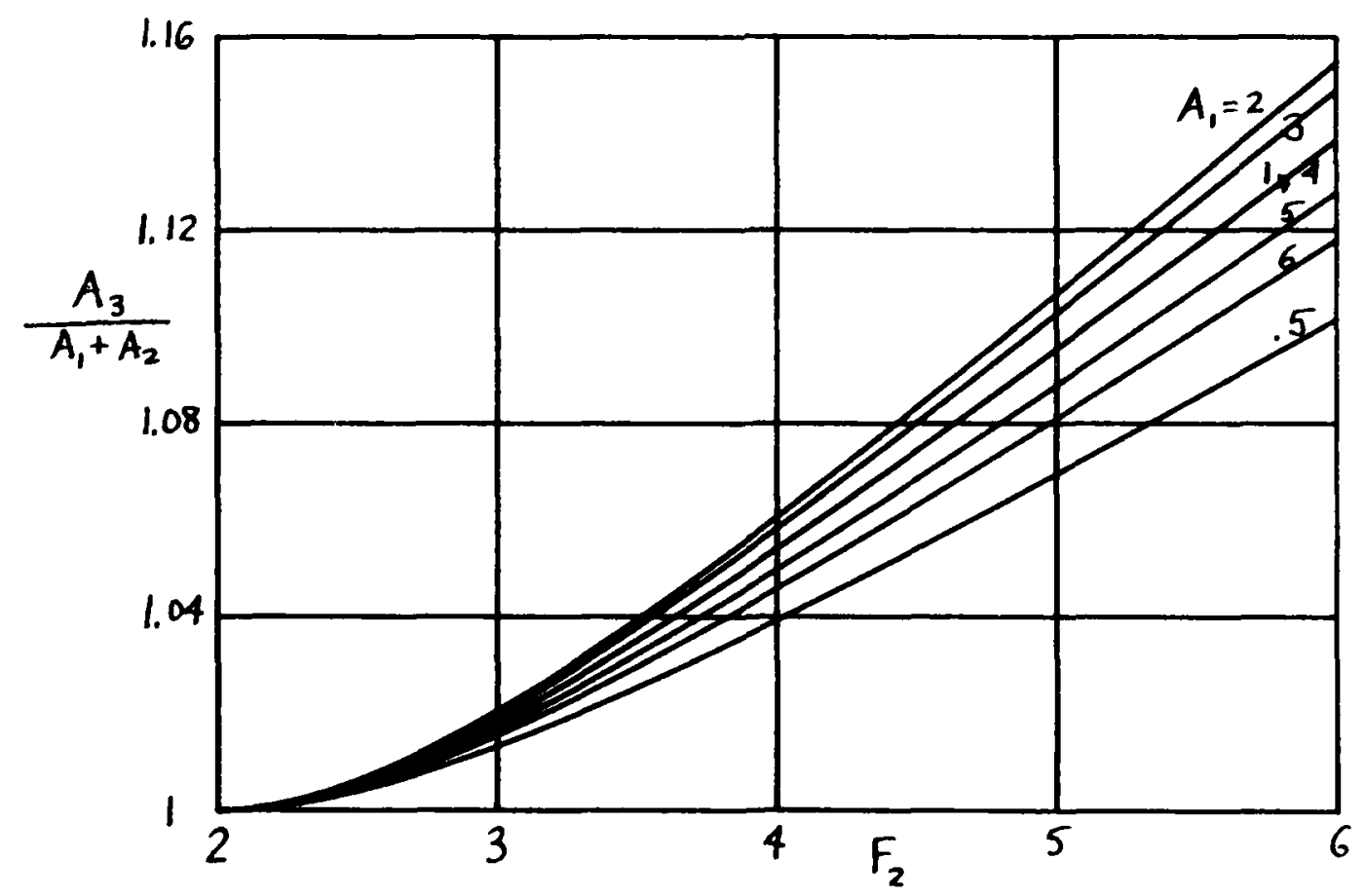

Figure 4. Area Ratio (34) for $F_{1}=2, A_{2}=2, \beta_{1}=\pi / 4, \beta_{2}=\pi / 4$ 


\section{SUMMARY}

The most compact WDF $W_{u}$ that can be used for two-dimensional smoothing of a measured WDF $W_{S}$ is a Gaussian function in two variables, when the measure of spread is quadratic in the time and frequency variables $t$ and $f$, or the reward measure is exponential. Furthermore, this two-dimensional convolution guarantees a non-negative modified distribution, since the result is equivalent to a short-term spectral estimate. Extensions to a particular class of generalized distributions yields the same optimum woF. The correspording waveform has Gaussian amplitude modulation and linear frequency-modulation.

The additional smearing caused by mismatched smoothing functions to the true parameters of a measured WDF has been investigated numerically for a few examples, and found not to be overly sensitive to the exact values. However, the multitude of parameters has prevented simplification of the area spread factor; accordingly, a program allowing calculation of particular cases is included to allow for further investigation.

The WDFs for the Hermite functions of order $n$ are given in closed form, in terms of a Laguerre polynomial of order $n$. This result is extended to cross-WDFs in appendix $A$; in this manner, we can investigate the WDF of an arbitrary waveform when expanded in a weighted sum of Hermite functions, including linear frequency-modulation. 
We want to find that WDF, $W_{u}(t, f)$, which is maximally concentrated about the origin in $t, f$ space, where the measure of reward for concentration is

$$
R(t, f)=\exp \left[-a^{2} t^{2}-4 \pi^{2} b^{2} f^{2}-4 \pi c t f\right], \quad a, b, c \text { real }
$$

Thus, the maximum reward occurs at the origin,

$$
R(0,0)=1 \text {. }
$$

and the contours of equal reward are ellipses in the $t, f$ plane. In order for $R(t, f)$ to decay to zero as $t$ and/or $f$ tend to infinity, we must have

$$
0>0 \text {, }
$$

where

$$
0=a^{2} b^{2}-c^{2}
$$

The reward value associated with WOF $W_{u}$ is the real quantity

$$
V=\iint d t d f R(t, f) W_{u}(t, f) \text {, }
$$

which we wish to maximize, where

$$
W_{u}(t, f)=\int d \tau \exp (-i 2 \pi f \tau) u\left(t+\frac{\tau}{2}\right) u^{\star}\left(t-\frac{\tau}{2}\right)
$$

in terms of weighting $u(t)$. We must constrain the volume of $w_{u}$, in order that $V$ in $(A-5)$ not tend to infinity as $u(t)$ is simply increased in level. Thus, we have integral constraint

$$
1=\iint d t d f w_{u}(t, f)=\int d t|u(t)|^{2}
$$




\section{ALILRNATIVE FORM FOR $V$}

If we substitute $(A-6)$ in $(A-5)$, there follows

$$
v=\iint d t d \tau r(t, \tau) u\left(t+\frac{\tau}{2}\right) u^{\star}\left(t-\frac{\tau}{2}\right),
$$

where

$$
r(t, \tau)=\int d f \exp (-i 2 \pi f \tau) R(t, f)
$$

A more useful alternative form for $(A-8)$ is

$$
V=\iint d x d y k(x, y) u(x) u^{\star}(y) \text {, }
$$

where kernel

$$
k(x, y)=r\left(\frac{x+y}{2}, x-y\right) .
$$

EIGF NFUNCTIONS OF $K$

In this and the following subsection, kernel $k$ is Hermitian, but otherwise arbitrary; it is not limited to form $(A-11)$ with $(A-9)$ and $(A-1)$. Suppose $\left\{\lambda_{n}\right\}$ and $\left\{0_{n}\right\}$ are the eigenvalues and eigenfunctions of kernel K; i.e.,

$$
\int d x k(x, y) b_{n}(x)=\lambda_{n} n_{n}(y) \text { for } n=0,1,2, \ldots \text {. }
$$

where $\lambda_{0} \geq \lambda_{1} \geq \lambda_{2} \cdots$, and

$$
\int d x \theta_{n}^{*}(x) \sigma_{m}(x)=\delta_{n m} .
$$


TR 8317

Then the kernel can be expanded according to

$$
K(x, y)=\sum_{n=0}^{\infty} \lambda_{n} \theta_{n}^{\star}(x) \theta_{n}(y) .
$$

Also, there follows immediately

$$
\iint d x d y k(x, y) \emptyset_{n}(x) \theta_{n}^{\star}(y)=\lambda_{n} .
$$

EXPANSION OF $U$

Suppose we expand weighting $u$ in a series of eigenfunctions of Hermitian kernel $\mathrm{K}$ :

$$
u(x)=\sum_{n=0}^{\infty} g_{n} \sigma_{n}(x) \text {, where } g_{n}=\int d x u(x) \theta_{n}^{\star}(x)
$$

Then general reward expression $V$ in $(A-10)$ becomes

$$
\begin{aligned}
& v=\int d y u^{\star}(y) \int d x k(x, y) \sum_{n=0}^{\infty} g_{n} g_{n}(x)= \\
& =\sum_{n=0}^{\infty} g_{n} \int d y u^{\star}(y) \lambda_{n} \theta_{n}(y)=\sum_{n=0}^{\infty}\left|g_{n}\right|^{2} \lambda_{n} .
\end{aligned}
$$

where we used $(A-12)$ and $(A-16)$. At the same time, the energy of $u$ in $(A-16)$ is

$$
E_{u}=\int d x|u(x)|^{2}=\sum_{n=0}^{\infty}\left|g_{n}\right|^{2}
$$


Now if the energy $E_{u}$ of $u$ is constrained at 1 , as in $(A-7)$, then the best choice of coefficients $\left\{g_{n}\right\}$ to maximize $V$ in $(i-17)$ is, since $\lambda_{0} \geq \lambda_{1} \geq \lambda_{2} \cdots$ obviously

$$
\left|g_{0}\right|=1 \text { and } g_{n}=0 \text { for } n \geq 1 \text {. }
$$

That is, the optimum weighting is

$$
u_{0}(x)=\emptyset_{0}(x) \exp (i \theta) \text {. }
$$

where constant $\theta$ is arbit.ary, while the maximum reward is

$$
V_{\max }=\lambda_{0} .
$$

That is, the zero-th order eigenvalue and eigenfunction of general Hermitian kernel $k$ in $(A-12)$ are the solutions to the problem of interest here, namely maximization of reward value $V$ in $(A-10)$ by choice of weighting $u$. For a gerieral kernel, a recursive numerical procedure could be employed on (A-12) to determine $\lambda_{0}$ and $\omega_{0}$. if desired.

The formulation in these last two subsections is actually general enough to cover the earlier penalty function considered in (3) et seq. The only difference is that the eigenvalues $\left\{\lambda_{n}\right\}$ now increase with $n$, and we must select the eigenfunction corresponding to the minimum eigenvalue, in order to realize the least penalty. This approach is the subject of appendix 0 . 


\section{SPECIAL CASE OF EXPONENTIAL REWARD}

We now specialize the general results of the previous two subsections to the reward function $(A-1)$. Substitution in $(A-9)$ and use of $(A-4)$ yields

$$
r(t, \tau)=\frac{1}{2 \sqrt{\pi} b} \exp \left[-\frac{Q t^{2}+\frac{1}{4} \tau^{2}-i c t \tau}{b^{2}}\right]
$$

compare $[1,(F-9)$ and $(F-12)]$. Then $(A-11)$ immediately gives Hermitian kerne?

$$
K(x, y)=\frac{1}{2 \sqrt{\pi} b} \exp \left[-\frac{x^{2} D^{\star}+y^{2} D+2 x y(Q-1)}{4 b^{2}}\right] \text {, }
$$

where

$$
D=0+1+i 2 c .
$$

At this point, we refer to Mehler's expansion $[3,(67)]$ to obtain (after some labor)

$$
K(x, y)=\sum_{n=0}^{\infty} \lambda_{n} \sigma_{n}^{\star}(x) \sigma_{n}(y)
$$

where

$$
\begin{gathered}
\lambda_{n}=\frac{(1-\sqrt{Q})^{n}}{(1+\sqrt{Q})^{n+1}}, \\
n_{n}(x)=A \exp \left[-\frac{x^{2}}{2} \frac{\sqrt{Q}+i c}{b^{2}}\right] \frac{1}{\sqrt{n !}} H e_{n}\left(2^{1 / 2} Q^{1 / 4} x / b\right) .
\end{gathered}
$$

and

$$
|A|^{2}=\frac{Q^{1 / 4}}{\sqrt{\pi} b}
$$


The function $\mathrm{He}_{n}(x)$ is the Hermite polynomial $[4,22.2 .15]$. It is easily verified that $(A-27)$ satisfies orthonormality relation $(A-13)$.

OPTIMUM WE IGHTING

Since $Q>0$ by $(A-3)$, the eigenvalues in $(A-26)$ satisfy $\lambda_{0}>\lambda_{1}>\lambda_{2} \cdots$. Therefore, the maximum reward is

$$
v_{\max }=\lambda_{0}=\frac{1}{1+\sqrt{0}}=\frac{1}{1+\sqrt{a^{2} b^{2}-c^{2}}},
$$

and the corresponding weighting is

$$
u_{0}(t)=\sigma_{0}(t)=A \exp \left[-\frac{t^{2}}{2} \frac{\sqrt{Q}+i c}{b^{2}}\right]
$$

from (A 27) and (A-28). This is identical to (20) combined with (11). Therefore the optimum WDF is again (22) for reward measure $(A-1)$, as well as penalty measure ( 3 ). The waveform in (A-30) has Gaussian amplitude modulation and linear frequency-modulation.

HIGHER-ORDER HERMIIE FUNCTIONS

For $n>0$, the reward values $\left\{\lambda_{n}\right\}$ in $(A-26)$ are all less than optimum value $\lambda_{0}$. We have succeeded in obtaining these explicit values without having to evaluate the WDFs of the corresponding Hermite waveforms in $(A-27)$. We now rectify this situation. The wOF of ${ }_{n}(t)$ in $(A-27)$ is given by integral 
TR 8317

$$
\begin{aligned}
& W_{n}(t, f)= \int d \tau \exp (-i 2 \pi f \tau) \theta_{n}\left(t+\frac{\tau}{2}\right) \theta_{n}^{\star}\left(t-\frac{\tau}{2}\right)= \\
&=\frac{|A|^{2}}{n !} \int d \tau \exp \left[-i 2 \pi f \tau-\frac{1}{2} B\left(t+\frac{\tau}{2}\right)^{2}-\frac{1}{2} B^{*}\left(t-\frac{\tau}{2}\right)^{2}\right] * \\
& * H_{n}\left(F\left(t+\frac{\tau}{2}\right)\right) H e_{n}\left(F\left(t-\frac{\tau}{2}\right)\right) .
\end{aligned}
$$

where

$$
B=\frac{\sqrt{Q}+i C}{b^{2}}, F=\frac{2^{1 / 2} Q^{1 / 4}}{b},|A|^{2}=\frac{Q^{1 / 4}}{\sqrt{\pi} b} .
$$

Now a more general integral result already exists in closed form; from [5, p. 292, (30)], we have, in a form more useful for present purposes,

$$
\begin{gathered}
\int d x \exp \left(-\frac{1}{2} x^{2}+a x\right) H e_{m}(b+x) H e_{n}(b-x)= \\
=\sqrt{2 \pi}(-1)^{m} m !(b-a)^{n-m} L_{m}^{(n-m)}\left(b^{2}-a^{2}\right) \exp \left(a^{2} / 2\right) \quad \text { for } m \leq n .
\end{gathered}
$$

where $L_{m}^{(\alpha)}(x)$ is the generalized Laguerre polynomial [4, 22.2.12]. When $(A-33)$ is used on $(A-32)$, there follows, for the WOF of waveform $\sigma_{n}(t)$ in (A-27), the compact result

$$
w_{n}(t, f)=(-1)^{n} 2 L_{n}(2 U) \exp (-U) \text {. }
$$

where

$$
u=\frac{a^{2} t^{2}+4 \pi^{2} b^{2} f^{2}+4 \pi c t f}{\sqrt{0}}
$$

This result reduces to (22) for $n=0$. Again, contours of equal values of the WOF are ellipses in the $t, f$ plane. 
TR 8317

CROSS-WOFS

Suppose a general waveform $u(t)$ is expanded in a set of orthonormal

Hermite functions with linear frequency-modulation ( $\alpha$ positive real, $B$ real)

$$
D_{n}(t)=\left(\frac{a}{n}\right)^{1 / 4} \exp \left[-\frac{1}{2}(\alpha+i \beta) t^{2}\right] H e_{n}(\sqrt{2 a} t) / \sqrt{n !} .
$$

according to

$$
u(t)=\sum_{n=0}^{\infty} u_{n} w_{n}(t) .
$$

Then the WOF of $u(t)$ becomes

$$
\begin{gathered}
w_{u}(t, f)=\int d \tau \exp (-i 2 \pi f \tau) u\left(t+\frac{\tau}{2}\right) u^{\star}\left(t-\frac{\tau}{2}\right)= \\
=\sum_{m, n=0}^{\infty} u_{m} u_{n}^{*} w_{m n}(t, f) .
\end{gathered}
$$

where cross-WDF

$$
W_{m n}(t, f)=\int d \tau \exp (-i 2 \pi f \tau) m_{m}\left(t+\frac{\tau}{2}\right) \omega_{n}^{\star}\left(t-\frac{\tau}{2}\right)
$$

When $(A-36)$ is substituted in $(A-39)$, and $(A-33)$ is utilized, the cross-WDF can be expressed as

$$
W_{m n}(t, f)=2(-1)^{m} \sqrt{\frac{m !}{n !}} z^{n-m} L_{m}^{(n-m)}\left(|z|^{2}\right) \exp \left(-|z|^{2} / 2\right) \text { for } m \leq n \text {. }
$$

$(A-40)$

where

$$
\begin{gathered}
z=\sqrt{\frac{2}{\alpha}}[\alpha t+i(2 \pi f+B t)], \\
|z|^{2}=\frac{2}{\alpha}\left[\left(\alpha^{2}+B^{2}\right) t^{2}+4 \pi^{2} f^{2}+4 \pi \beta t f\right] .
\end{gathered}
$$

25 
TR 8317

These results generalize $[6, p p .456-7]$ and $[7, p .547]$.

The origin value of $(A-40)$ is

$$
w_{m n}(0,0)=2(-1)^{m} s_{m n} .
$$

consistent with unit energy of $\emptyset_{n}(t)$ and their even or odd character. The cross-WDF in $(A-40)$ is a function only of the three variables $m, n, z$, where $z$ is the complex rombination in (A-41). The parameters $a$ and $B$ in $(A-36)$ are perfectly general; when they are specialized to match $(A-27)$, and when we set $m=n$, then $(A-40)$ reduces to $(A-34)$. Equations $(A-38)$ and $(A-40)$ afford a direct calculation of the WDF of a general waveform $u(t)$, once the coefficients are determined by

$$
u_{n}=\int d t u(t) \sigma_{n}^{\star}(t)
$$




\section{APPENOIX B. MOMENTS OF OISTRIBUTION D}

The generalized smoothing distribution $D$ is given by (23) in terms of a double Fourier transform of product

$$
P(v, \tau)=\chi_{u}(v, \tau) a_{2}(v, \tau)
$$

Therefore, the inverse relation is

$$
P(v, \tau)=\iint d t d f \exp (-12 \pi v t+12 \pi f \tau) D(t, f) .
$$

If we let superscript $v$ denote a partial derivative with respect to $v$, there immediately follows from $(B-2)$.

$$
\begin{gathered}
P(0,0)=\iint d t d f D(t, f) \\
P^{v}(0,0)=-i 2 \pi \iint d t d f t D(t, f) \\
P^{\tau}(0,0)=i 2 \pi \iint d t d f f D(t, f) \\
P^{v v}(0,0)=-4 \pi^{2} \iint d t d f t^{2} D(t, f) \\
P^{v \tau}(0,0)=4 \pi^{2} \iint d t d f t f D(t, f) \\
p^{\tau \tau}(0,0)=-4 \pi^{2} \iint d t d f f^{2} D(t, f) .
\end{gathered}
$$

When these relations are written out explicitly in terms of $\chi_{u}$ and 
TR 8317

$q_{2}$, according to $(B-1)$, we find that the moments of $D$ are

$$
\begin{aligned}
& \iint d t d f D(t, f)=\chi_{u}(0,0) a_{2}(0,0) \\
& \iint d t d f t D(t, f)=\frac{i}{2 \pi}\left[x_{u}^{v}(0,0) a_{2}(0,0)+\chi_{u}(0,0) a_{2}^{v}(0,0)\right] \\
& \iint d t d f f D(t, f)=\frac{-1}{2 \pi}\left[\chi_{u}^{\tau}(0,0) a_{2}(0,0)+\chi_{u}(0,0) q_{2}^{\tau}(0,0)\right] \\
& \iint d t d f t^{?} D(t, f)=-\frac{1}{4 \pi^{2}}\left[\chi_{u}^{v v}(0,0) a_{2}(0,0)+2 \chi_{u}^{v}(0,0) q_{2}^{v}(0,0)+\right. \\
& \left.+\chi_{u}(0,0) q_{2}^{v v}(0,0)\right] \\
& \iint d t d f t f D(t, f)=\frac{1}{4 \pi^{2}}\left[x_{u}^{v \tau}(0,0) a_{2}(0,0)+x_{u}^{\tau}(0,0) a_{2}^{v}(0,0)+\right. \\
& \left.+\chi_{u}^{v}(0,0) q_{2}^{\tau}(0,0)+\chi_{u}(0,0) q_{2}^{v \tau}(0,0)\right] \\
& \iint d t d f f^{2} D(t, f)=-\frac{1}{4 \pi^{2}}\left[X_{u}^{\tau \tau}(0,0) a_{2}(0,0)+2 \chi_{u}^{\tau}(0,0) a_{2}^{\tau}(0,0)+\right. \\
& \left.+x_{u}(0,0) q_{?}^{\tau \tau}(0,0)\right] .
\end{aligned}
$$

Since $a_{2}$ is a double fourier transform of $V_{2}$, of exactly the same form as $(B-2)$, it follows immediately, by similarity to $(B-3)$, that the required derivatives of $a_{2}$ in $(B-4)$ can be found from smoothing function $v_{2}$ as 
TR 8317

$$
\begin{gathered}
a_{2}(0,0)=\iint d t d f v_{2}(t, f) \\
a_{2}^{v}(0,0)=-i 2 \pi \iint d t d f t v_{2}(t, f) \\
a_{2}^{\tau}(0,0)=i 2 \pi \iint d t d f f v_{2}(t, f) \\
q_{2}^{v v}(0,0)=-4 \pi^{2} \iint d t d f t^{2} v_{2}(t, f) \\
q_{2}^{v \tau}(0,0)=4 \pi^{2} \iint d t d f t f v_{2}(t, f) \\
q_{2}^{\tau \tau}(0,0)=-4 \pi^{2} \iint d t d f f^{2} v_{2}(t, f) .
\end{gathered}
$$

COMPLEX AMBIGUITY FUNCTION PROPERTIES

The required derivatives of $\chi_{u}$ in $(B-4)$ can be determined from definition (24). We list them here for completeness and future reference:

$$
\begin{gathered}
\chi_{u}(0,0)=\int d t|u(t)|^{2} \\
\chi_{u}^{v}(0,0)=-i 2 \pi \int d t t|u(t)|^{2} \\
\chi_{u}^{\tau}(0,0)=i \int d t \operatorname{Im}\left\{u^{\prime}(t) u^{\star}(t)\right\} \\
\chi_{u}^{v v}(0,0)=-4 \pi^{2} \int d t t^{2}|u(t)|^{2} \\
\chi_{u}^{v \tau}(0,0)=2 \pi \int d t \operatorname{Im}\left\{u^{\prime}(t) u^{\star}(t)\right\} \\
\chi_{u}^{\tau \tau}(0,0)=-\int d t\left|u^{\prime}(t)\right|^{2} .
\end{gathered}
$$

29 
TR 8317

These quantities are all real, with the exception of the two single derivatives, both of which are purely imaginary. These second-order derivative values of $\chi_{v}$ can be expressed solely in terms of $u(t)$ and $u^{\prime}(t)$.

Since we can express complex ambiguity function $\chi_{u}$ in terms of the WDF $W_{u}$ according to

$$
\chi_{u}(v, \tau)=\iint d t d f \exp (-i 2 \pi v t+i 2 \pi f \tau) w_{u}(t, f) .
$$

it readily follows from $(B-6)$ that

$$
\begin{gathered}
\iint d t d f w_{u}(t, f)=\int d t|u(t)|^{2} \\
\iint d t d f t w_{u}(t, f)=\int d t t|u(t)|^{2} \\
\iint d t d f f w_{u}(t, f)=\frac{1}{2 \pi} \int d t \operatorname{Im}\left\{u^{\prime}(t) u^{\star}(t)\right\} \\
\iint d t d f t^{2} w_{u}(t, f)=\int d t t^{2}|u(t)|^{2} \\
\iint d t d f t w_{u}(t, f)=\frac{1}{2 \pi} \int d t \operatorname{Im}^{2}\left\{u^{\prime}(t) u^{\star}(t)\right\} \\
\iint d t d f f^{2} W_{u}(t, f)=\frac{1}{4 \pi^{2}} \int d t\left|u^{\prime}(t)\right|^{2} .
\end{gathered}
$$

30 


\section{APPENOIX C. GENERAL TILTED ELLIPSE}

It will be convenient to be able to specify the area, tilt, and shape factor of an ellipse directly, instead of trying to solve for these quantities from the general form

$$
\frac{1}{2} a x^{2}+\frac{1}{2} b y^{2}+\sqrt{a b} \rho x y=1
$$

employed in $[1,(J-2)]$. Accordingly, as done in [1, app. 0$]$, we employ the rotated coordinates depicted in figure $\mathrm{C}-1$ below. The equation of the ellipse in $x^{\prime}, y^{\prime}$ space is

$$
\left(\frac{x^{\prime}}{x_{0}^{\prime}}\right)^{2}+\left(\frac{y^{\prime}}{y_{0}^{\prime}}\right)^{2}=1 .
$$

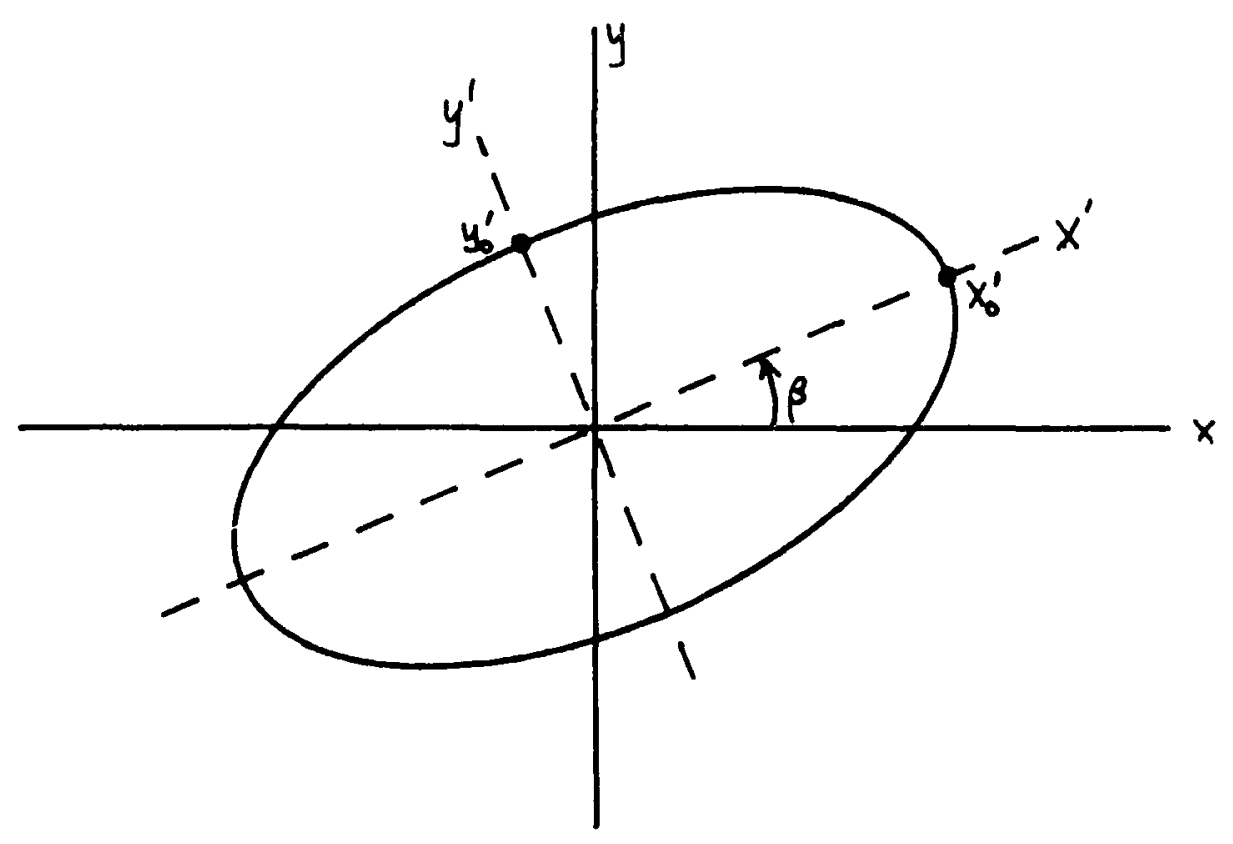

Figure $\mathrm{C}-\mathrm{T}$. Rotated Coordinate Axes 
TR 8317

But since the area of this ellipse is

$$
A=\pi x_{0}^{1} y_{0}^{\prime},
$$

while its shape factor is

$$
F=\frac{x_{0}^{\prime}}{y_{0}^{\prime}}
$$

it is a simple matter to find that

$$
A F=\pi x_{0}^{\prime 2}, A / F=\pi y_{0}^{\prime 2},
$$

leading to the desirable form

$$
\frac{x^{\prime 2}}{F}+F y^{\prime 2}=\frac{A}{\pi} .
$$

Furthermore, the coordinate axes in figure $\mathrm{C}-1$ are related according to

$$
\left.\begin{array}{l}
x^{\prime}=x C+y S \\
y^{\prime}=-x S+y C
\end{array}\right\} \quad C=\cos (\beta), S=\sin (\beta) \text {. }
$$

Substitution in $(C-6)$ yields

$$
\frac{1}{2} x^{2}\left(\frac{C^{2}}{F}+F S^{2}\right)+\frac{1}{2} y^{2}\left(\frac{S^{2}}{F}+F C^{2}\right)+x y S C\left(\frac{1}{F}-F\right)=\frac{A}{2 \pi}
$$

which is of the form $(C-1)$ under identifications

$$
\begin{aligned}
& a=\frac{2 \pi}{A}\left(\frac{C^{2}}{F}+F S^{2}\right), \quad b=\frac{2 \pi}{A}\left(\frac{S^{2}}{F}+F C^{2}\right), \\
& \rho=\frac{r}{\sqrt{1+r^{2}}} \text { with } \quad Y=S C\left(\frac{1}{F}-F\right) .
\end{aligned}
$$

Once area $A$, tilt $B$, and shape factor $F$ are specified, $(C-9)$ affords a ready calculation of $a, b, \rho$; quantities $C$ and $S$ are given by $(C-1)$. Since 
$\rho=\sin (\theta)$ in $[1,(J-6)]$, we have

$$
\sin (\theta)=\frac{\gamma}{\sqrt{1+\gamma^{2}}}, \quad \cos (\theta)=\frac{1}{\sqrt{1+\gamma^{2}}},
$$

which are needed below.

In order to distinguish the two Gaussian mountains being doubly convolved in $[1,(J-2)]$, we label them with subscripts 1 and 2 , respectively, thereby obtaining

$$
\begin{gathered}
a=\frac{2 \pi}{A_{1}}\left(\frac{C_{1}^{2}}{F_{1}}+F_{1} S_{1}^{2}\right), b=\frac{2 \pi}{A_{1}}\left(\frac{S_{1}^{2}}{F_{1}}+F_{1} C_{1}^{2}\right), S_{1}=\sin \left(B_{1}\right), C_{1}=\cos \left(B_{1}\right), \\
r_{1}=S_{1} C_{1}\left(\frac{1}{F_{1}}-F_{1}\right), \sin (\theta)=\frac{r_{1}}{\sqrt{1+r_{1}^{2}}}, \cos (\theta)=\frac{1}{\sqrt{1+r_{1}^{2}}},(C-1)
\end{gathered}
$$

and

$$
\begin{aligned}
& c=\frac{2 \pi}{A_{2}}\left(\frac{C_{2}^{2}}{F_{2}}+F_{2} S_{2}^{2}\right), d=\frac{2 \pi}{A_{2}}\left(\frac{S_{2}^{2}}{F_{2}}+F_{2} C_{2}^{2}\right), S_{2}=\sin \left(B_{2}\right), C_{2}=\cos \left(B_{2}\right), \\
& r_{2}=S_{2} C_{2}\left(\frac{1}{F_{2}}-F_{2}\right), \sin (\theta)=\frac{r_{2}}{\sqrt{1+r_{2}^{2}}} \cdot \cos (\theta)=\frac{1}{\sqrt{1+r_{2}^{2}}} .
\end{aligned}
$$

We are now in a position to evaluate the effective area $A_{3}$ of the resultant convolution; namely from $[1,(3-9)-(J-11)]$, we have

$$
\frac{A_{3}}{A_{1}+A_{2}}=\frac{\sqrt{D}}{\sqrt{a b} \cos (\theta)+\sqrt{c d} \cos (\theta)} \text {, }
$$


TR 8317

where

$$
D=a b \cos ^{2}(\theta)+c d \cos ^{2}(\theta)+a d+b c-2 \sqrt{a t c d} \sin (\theta) \sin (\theta) \cdot(c-14)
$$

The minimum value of $(C-13)$ is 1 , attained when shape factors $F_{1}=F_{2}$ and tilts $B_{1}=\beta_{2}$. More generally, when we specify

$$
\begin{aligned}
& A_{1}, B_{1}, F_{1} \text { for ellipse } 1, \\
& A_{2}, B_{2}, F_{2} \text { for ellipse } 2 \text {, }
\end{aligned}
$$

equations $(C-11)$ and $(C-12)$ allow for evaluation of all the parameters

needed in $(C-13)$ and $(C-14)$. A sample program in BASIC is attached.

Subroutine $E$ computes $a, b, \sin (\theta), \cos (\theta)$ as given by $(C-9)$ and $(C-10)$ in

terms of given area $A$, shape factor $F$, and tilt $B(=B)$. 
EIHIT

FLOTTEF: I

GFFFHICS DN

WIHIMW $-F I+4, F I 4,1,1.25$

DFIII FI $8, .05$

$F 2=2$

$E 1=F 1 \cdot 4$

$\mathrm{AZ}=\mathrm{Z}$

SHAFE FALTOK

$\mathrm{F} Z=z$

TILT

DATH

AREA

$5,1,2,3,4,5,0$

IJ I H FI, $1: ?$

FEAI HI: *

FOR $I=1 \quad T Q \quad \vec{T}$

$\bar{H} 1=\bar{H} 1: I$,

CHLL EIAI, F1, E1, HE, ES, St, C.)

$\bar{A} B=\bar{H} \equiv \pm B \equiv$

FIF $E Z=-F I+T O F I+S T E F$ FI 101

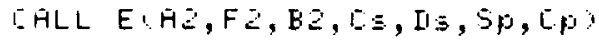

C. $d=I_{-} \equiv * \mathrm{I} \equiv$

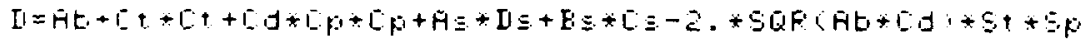

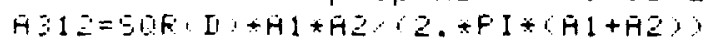

FLOT BQ, $\mathrm{HO} 1 \mathrm{Z}$

HE:TT B2

FEHIIF

HE.:T I

FHISEE

ENI

SUE E, H, F, E, $A S, B S, S t, C t)$

$S=S 1+i \mathrm{E}$.

$E=0,0, E$

$\square=5+[* 1, F-F)$

$E A=50 R(1 .+5 * I)$

$S+=5 \cdot 5$

$i=1$. 50

$i z=1+i$

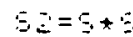

$T=2 . P I A$

$\bar{H} \equiv=T * 1, \mathrm{~T} Z \mathrm{~F}+\mathrm{S} Z \mathrm{LF}$,

$E \equiv=T+C S 2 \cdot \mathrm{F}+122 * \mathrm{~F}$,

SIJEEHIJ 


\section{APPENDIX D. KERNEL APPROACH TO PENALTY FUNCTION}

The general formulation in $(A-11)$ through $(A-21)$ will be applied in this appendix to the penalty function (3):

$$
P(t, f)=a^{2} t^{2}+4 \pi^{2} b^{2} f^{2}+4 \pi c t f .
$$

Substitution in $(A-9)$ (in place of reward $R$ ) yields

$$
\begin{aligned}
r(t, \tau) & =\int d f \exp (-i 2 \pi f \tau) P(t, f)= \\
& =\int d f \exp (-i 2 \pi f \tau)\left(a^{2} t^{2}+4 \pi^{2} b^{2} f^{2}+4 \pi c t f\right)= \\
& =a^{2} t^{2} \delta(\tau)-b^{2} \delta^{\prime \prime}(\tau)+i 2 c t \delta^{\prime}(\tau) .
\end{aligned}
$$

Then kernel $K$ follows from $(A-11)$ as

$$
\begin{gathered}
K(x, y)=r\left(\frac{x+y}{2}, x-y\right)= \\
=\frac{a^{2}}{4}(x+y)^{2} \delta(x-y)-b^{2} \delta^{\prime \prime}(x-y)+i c(x+y) \delta^{\prime}(x-y) .
\end{gathered}
$$

which is Hermitian.

The integral equation $(A-12)$, that must be solved, can be simplified by use of the facts that

$$
\begin{gathered}
\frac{1}{4} a^{2} \int d x(x+y)^{2} \delta(x-y) \emptyset_{n}(x)=a^{2} y^{2} \emptyset_{n}(y), \\
-b^{2} \int d x \delta^{\prime \prime}(x-y) \emptyset_{n}(x)=-b^{2} \sigma_{n}^{\prime \prime}(y) . \\
\text { ic } \int d x(x+y) \delta^{\prime}(x-y) \sigma_{n}(x)=-i c\left[2 y \sigma_{n}^{\prime}(y)+\sigma_{n}(y)\right] .
\end{gathered}
$$


where the last two results are obtained by integration by parts. Then $(A-12)$ yields differential equation

$$
b^{2} n_{n}^{\prime \prime}(y)+i 2 c y 0_{n}^{\prime}(y)+\left(\lambda_{n}+i c-a^{2} y^{2}\right) b_{n}(y)=0 .
$$

If we try solution

$$
\sigma_{0}(y)=A \exp \left(-\frac{1}{2} b_{y}^{2}\right)
$$

in $(0-5)$, we find it to be acceptable if we take

$$
B=\frac{\sqrt{a}+i c}{b^{2}}, \quad \lambda_{0}=\sqrt{a} .
$$

These results agree with (11) and (20), as expected. To find the general solution of $(D-5)$, we try solution form

$$
0(y)=\exp \left(-\frac{1}{2} B y^{2}\right) H(y) .
$$

with $B$ still given by $(D-7)$. This form in $(D-8)$ is no loss of generality since $H$ is still arbitrary. Use of $(0-8)$ in $(0-5)$ results in

$$
\begin{gathered}
b^{2} H^{\prime \prime}(y)+2 y H^{\prime}(y)\left(-b^{2} B+i c\right)+ \\
+H(y)\left(-b^{2} B+b^{2} B^{2} y^{2}-i 2 c B y^{2}+\lambda+i c-a^{2} y^{2}\right)=0 .
\end{gathered}
$$

When the value for $B$ in $(0-7)$ is utilized, $(0-9)$ simplifies to

$$
b^{2} H^{\prime \prime}(y)-2 \sqrt{0} \text { y } H^{\prime}(y)+(\lambda-\sqrt{0}) H(y)=0 .
$$

(As a partial check, if $H(y)=A$, then $\lambda=\sqrt{0}$, as in $(0-7)$.)

Now, in $(0-9)$, let

$$
H(y)=G(F y), H^{\prime}(y)=F G^{\prime}(F y), H^{\prime \prime}(y)=F^{2} G^{\prime \prime}(F y),
$$


TR 8317

where $F$ is arbitrary for the moment, thereby obtaining

$$
b^{2} F^{2} G^{\prime \prime}(F y)-2 \sqrt{0} \text { y } F G^{\prime}(F y)+(\lambda-\sqrt{0}) G(F y)=0 .
$$

Now let $x=F y$ to get

$$
G^{\prime \prime}(x)-\frac{2 \sqrt{Q}}{b^{2} F^{2}} \times G^{\prime}(x)+\frac{\lambda-\sqrt{Q}}{b^{2} F^{2}} G(x)=0 .
$$

If we now let (without loss of generality)

$$
F=\frac{2^{1 / 2} Q^{1 / 4}}{b}
$$

then $(0-13)$ simplifies further to

$$
G^{\prime \prime}(x)-x G^{\prime}(x)+\frac{\lambda-\sqrt{Q}}{2 \sqrt{0}} G(x)=0 .
$$

We now appeal to $[4,22.6 .21]$ and observe that if

$$
\frac{\lambda-\sqrt{Q}}{2 \sqrt{Q}}=n=\text { integer } .
$$

then a solution of $(D-15)$ is

$$
G(x)=H e_{n}(x), \lambda_{n}=\sqrt{Q}(1+2 n) .
$$

Also, $(0-11)$ yields

$$
H(y)=G(F y)=H e_{n}(F y) \text {. }
$$

While (0-8) gives

$$
\sigma_{n}(y)=A \exp \left(-\frac{1}{2} B y^{2}\right) H e_{n}(F y) / \sqrt{n !} \text {. }
$$

with

$$
B=\frac{\sqrt{Q}+i c}{b^{2}}, F=\frac{2^{1 / 2} Q^{1 / 4}}{b},|A|^{2}=\frac{Q^{1 / 4}}{\sqrt{\pi b}} .
$$

38 
where the unit energy normalization of ${ }_{n}$ has been imposed. The corresponding eigenvalue follows from $(0-17)$ as

$$
\lambda_{n}=\sqrt{0}(1+2 n)=\sqrt{a^{2} b^{2}-c^{2}}(1+2 n) .
$$

The minimum obviously occurs for $n=0$.

Result $(D-19)$ agrees with $(A-27)$. However, the $\lambda_{n}$ given here by $(0-21)$ differs from that given by $(A-26)$, because we are solving for the minimum penalty here versus the maximum reward there. 
TR 8317

\section{REFERENCES}

1. A. H. Nutta 11, Wigner Distribution Function: Relation to Short-Term Spectral Estimation, Smoothing, and Performance in Noise, NUSC Technical Report 8225, Naval Underwater Systems Center, New London, CT, 16 February 1988.

2. L. Cohen and T. E. Posch, "Positive Time-Frequency Distribution Functions," IEEE Transactions on Acoustics, Speech, and Signal Processing, vol. ASSP-33, no. 1, PD. 31-38, February 1985.

3. J. F. Barrett and D. G. Lampard, "An Expansion for Some Second-Order Probability Oistributions and its Application to Noise Problems," IRE Iransactions on Information Theory, volume IT-1, number 1, pp. 10-15, March 1955.

4. Handbook of Mathematical Functions, National Bureau of Standards, Applied Mathematics Series, number 55, U.S. Department of Commerce, u.S. Goveinment Printing Offirs, Washington, D.C., June 1964.

5. A. Erdëlyi et al, Tables of Integral Transforms, volume II, McGraw-Hill Book Company, NY, 1954.

6. H. J. Groenewold, "On the Principles of Elementary Quantum Mechanics," Physica, vol. XII, no. 7, pr. 405-460, October 19 r.6.

7. M. S. Bartlett and J. E. Moyal, "The Exact Transition Probabilities of Quantum-Mechanical Oscillators, Calculated by the Phase-Space Method," Proc. of Cambridge Phil. Soc.. vol, 45, pp. 545-553, 1949. 
INIITAL DISTRIBUTION LIST

Addressee

No. of Copies

ADMIRALTY RESEARCH ESIABLISHMENT, LONDON, ENGLAND

(Or. L. Lloyd)

APPI IED PHYSICS LAB, JOHN HOPKINS (John C. Stapleton)

APPI.IED PHYSICS LAB, U. WASHINGTON (C. Eggan)

Contract N00024-85-C-6264

APPLIEO RESEARCH LAB, PENN STATE, (Dr. D. Ricker)

Contract NO0024-85-C-6041

APPLIED RESEARCH LAB, U. TEXAS (Or. M. Frazer)

Contract NOD024-86-C-6134-4-2-3

A \& T, STONINGTON, CT ( $H$. Jarvis)

APPLIED SEISMIC GROUP, (R. LaCOSS)

ASST SEC NAV

ASTRON RESEARCH \& ENGR, SANTA MONICA, CA (Dr. A. Piersol)

AUSTRALIAN NATIONAL UNIV. CANBERRA, AUSTRALIA

(Prof. B. Anderson)

BBN, Arlington, Va. (Or. H. Cox)

BBN, Cambridge, MA (H. Gish)

$B B N$, New London, Ct. (Dr. P. Cable)

BELL COMMUNICATIONS RESEARCH, Morristown, NJ (J. Kaiser and D. Sunday (Library)

BENDAT, JULIUS DR., 833 Moraga Dr. . LA, CA

DR. Norman Bleistein, Denver, CO 80222

CANBERRA COLLEGE OF ADV. EDUC, BELCONNEN, A.C.T.

CNA

AUSTRALIA (P. Morgan)

COAST GUARD ACADEMY, New London, CT (Prof. J. Wolcin)

COAST GUARD R \& D, Groton, CT (Library)

COGENT SYSTEMS, INC, (J. Costas)

CONCOROIA UNIVERSITY H-915-3, MONTREAL, QUEBEC CANADA

(Prof. Jeffrey Krolik)

CNO (NOP-098)

CNR-OCNR-00, 10, 11, 12, 13, 20 (2),122, 123, 124

DTNSRDC, BETHESDA

DAVIO W. TAYLOR RESEARCH CNTR, ANNAPOLIS, MD

(P. Prendergast, Code 2744)

DAVIO W. TAYLOR RESEARCH CNTR, BETHESDA, MD

(N. Zimmerman, Code 5220)

DARPA, ARLINGTON, VA (A. Ellinthorpe)

DALHOUSIE UNIV., HALIFAX, NOVA SCOTIA, CANADA (Dr. B. RUddiCK)

DEFENCE RESEARCH ESTAB. ATLANTIC, DARTMOUTH, NOVA SCOTIA (Library)

DEFENCE RESEARCH ESTAB. PACIFIC, VICTORIA, CANADA

(Dr. D. Thomson)

DEFENCE SCIENTIFIC ESTABLISHMENT, MINISTRY OF DEFENCE, AUCKLAND, N Z. (Dr. L. Hall)

DEFENSE SYSTEMS, INC, MC LEAN, VA (Or. G. Sebestyen)

DIA

DTIC

DTNSRDC

OREXEL UNIV, (Prof. S. Kesler)

EDO CORP, College Point, NY (M. Blanchard)

NICRAD 87-NUSC-029 of 23 July 1987 
INIITAL DISTRIBUTION LIST

EG\&G, Manassas, VA (Dr. J. Hughen and D. Frohman) GENERAL ELECTRIC CO. PITTSFIELO, MA (Mr. R. RaCe) GENERAL ELECTRIC CO, SYRACUSE, NY (Mr. J. L. ROgerS) GENERAL ELECTRIC CO, ( 0 . Winfield) N00024-87-C-6087) HAHN, WM, Apt. 701, 500 23rd St. NW, Wash, DC 20037 HARRIS SCIENTIFIC SERVICES, DObDS Ferry, NY (B. Harris) HUGHES AIRCRAFT, Fullerton, CA (S. Autrey) HUGHES AIRCRAFT, Buena Park, CA (T. Posch) IBM, Manassas, VA (G. Demuth)

INDIAN INSTITUTE OF SCIENCE, BANGALSORE, INOIA (N. Srinivasa) JOHNS HOPKINS UNIV, LAUREL, MD (J. C. Stapleton) LINCOM CORP., NORTHBORO, MA 01532 (Dr. T. Schonhoff) MAGNAVOX GOV \& IND ELEC CO, Ft. Wayne, IN (R. KenefiC) MARINE BIOLOGICAL LAB, Woods Hole, MA

MARINE PHYSICAL LABORATORY SCRIPPS

MARTIN MARIETTA BALTIMORE AEROSPACE, Baltimore, MD

(S. Lawrence Marple)

MASS. INSTITUTE OF TECHNOLOGY (Prof. A. Baggaroer)

MBS SYSTEMS, NORWALK, CT (A. Winder)

MIODLETON, DAVID, $127 \mathrm{E}$. 91st ST, NY, NY

NADC (5041, M. Mele)

NAIR-03

NASC, NAIR $-00,03$

NATIONAL RADIO ASTRONOMY OBSERVATORY (F. SChWAb)

NATO SACLANT ASW RESEARCH CENTRE, APO NY, NY 09019 (Library

R. E. Sullivan and G. Tacconi)

NAVAIR (03, PMA 264)

NAVAL INTELLIGENCE COMMAND

NAVAL INTELLIGENCE SUPPORT CTR

NRL UND SOUND REF DET, ORLANDO, FL

NAVAL OCEANOGRAPHIC OFFICE

NAVAL SEA SYSTEMS COMMAND-SEA-00, 63, 630, PMS-409, PMS-11

NAVAL SYSTEMS DIV., SIMRAD SUBSEA A/S, NORWAY (E. B. Lunde) NCSC

NICHOLS RESEARCH CORP., Wakefield, MA (T. Marzetta)

NOROA (Or. B. Adams)

NOROA (Code 345) N STL Station, MS 39529

NORTHEASTERN UNIV. (Prof. C. L. Nikias)

NORWEGIAN DEFENCE RESEARCH EST, NORWAY (Or J. Glattetre)

NOSC, (C. Sturdevant; 73, J. Lockwood, F. Harris, 743,

R. Smith; 62, R. Thuleen)

NPRDC

NPS, MONTEREY, CA (C. W. Therrien, Code 62 Ti)

NRL, Washington, DC (Dr. J. Buccaro, Dr. E. Franchi,

Dr. P. Abraham, Code 5132, A. A. Gerlach, W. Gabriel

(Code 5370), and $N$. Yen (Code 5135)

NRL, Arlington, VA (N. L. Gerr, Code 1111)

NSWC

NSWC DET FT. L.AUDERDALE

NSWC WHITE OAK LAB

NUSC DET FT. LAUDEROALE 
INIITAL DISTRIBUTION LIST

Addressee

No. of Copies

NUSC DET TUDOR HILL

NUSC DET WEST PALM BEACH (Dr. R. Kennedy Code 3802)

NWC

ORI CO, INC, New London, CT (G. Assard)

PENN STATE UNIV., State College, PA (F. Symons)

POHLER, R., 1034 Evergreen St., San Diego, CA 92106

PROME THEUS, INC, Sharon, MA (Dr. J. Byrnes)

PSI MARINE SCIENCES, New London, Ct. (Dr. R. Mellen)

PRICE, Di. Robert, $80 \mathrm{Hill}$ St., Lexington, Ma 02173

RAISBECK, Dr. Gordon, P.0. Box 4311, Portland, ME 04101

RAN RESEARCH LAB, DARLINGHURST, AUSTRALIA

RAYTHEON CO, Portsmouth, RI (J. Bartram, R. Connor)

and $S$. S. Reese) NICRAD 87-NUSC 013

RICHTER,W. 7615 Heritage Dr., Annandale, VA.

ROCKWELL INTERNATIONAL CORP, Anaheim, CA (L. Einstein and or. D. Elliott)

ROYAL MILITARY COLLEGE OF CANADA, (Prof. Y. Chan)

RUTGERS UNIV., Piscataway, NJ (Prof. S. Orfanidis)

RCA CORP, Moorestown, NJ (H. Upkowitz)

SAIC, Falls Church, VA (Or. P. Mikhalevsky)

SAIC, New London, CT (Dr. F. Dinapoli)

SANDIA NATIONAL LABORATORY (J. Claasen)

SCRIPPS INSTITUTION OF OCEANOGRAPHY

SE-00-, -63, 63-0, PMS-409, -411

SONAR \& SURVEILLANCE GROUP, DARLINGHURST, AUSTRALIA

SOUTHEASTERN MASS. UNIV (PrOF. C. H. Chen)

SPERRY CORP, GREAT NECK, NY

SPWAR-05, PDW 124

STATE UNIV. OF NY AT STONY BROOK (Prof. M. Barkat)

TEL-AVIV UNIV, TEL-AVIV, ISRAEL (Prof. E. Winstein)

TRACOR, INC, Austin, TX (Dr. T Leih and J. Wilkinson)

TRW FEDERAL SYSTEMS GROUP (R. Prager)

UNDERSEA ELECTRONICS PROGRAMS DEPT, SYRACUSE, NY (J. ROgerS)

UNIV. OF ALBERTA, EDMONTON, ALBE?TA, CANADA (K. Yeung)

UNIV OF CA, San Diego, CA (Prof. C. Helstrom)

UNIV. OF CT, Storrs, CT. (Library and Prof. C. Knapp)

UNIV OF FLA, GAINESVILLE, FL (D. Childers)

UNIV OF MICHIGAN, Cooley Lab, Ann Arbor, MI (Prof T. Birdsall)

UNIV. OF MINN, Minneapolis, Mn (Prof. M. Kaveh)

UNIV. OF NEWCASTLE, NEWCASTLE, NSW, CANADA (Prof. A. Cantoni)

UNIV. OF RI, Kingston, RI (Library, Prof. S. Kay,

Prof. L. Scharf, and Prof. D. Tufts)

UNIV. OF SOUTHERN CA., LA. (Dr. A. Polydoros PHE 414)

UNIV. OF STRATHCLYDE, ROYAL COLLEGE, Glasgow, Scotland

(Prof. T. Durrani)

UNIV. OF TECHNOLOGY, Loughborough, Leicestershire, England

(Prof. J. Griffiths)

UNIV. OF WASHINGTON, Seattle (Prof. D. Lyt?e)

URICK, ROBERT, Silver Springs, MD

VAN ASSELT, HENRIK, USEA S.P.A., LA SPEZIA, ITALY

WERBNER, A., $60 \mathrm{Elm}$ St., Medford, MA 02155 
INIITAL DISTRIBUTION LIST

Addressee

WESTINGHOUSE ELEC. CORP, WALTHAM, MA (D. Bennett)

WESTINGHOUSE ELEC. CORP, OCEANIC DIV, ANNAPOLIS, MO

(Or. H. L. Price and H. Newman N00024-87-C-6024)

WILSON ARTIC RESEARCH, INC.

WOODS HOLE OCEANOGRAPHIC INSTITUTION (Dr. R. Spindel and $\mathrm{Dr}$. E. Weinstein)

YALE UNIV. (Library, Prof. P. Schultheiss and Prof. F. Tuteur)
No. of Copies 